\title{
LAS EVALUACIONES ECONÓMICAS EN SALUD: HACIA UN ACERCAMIENTO ENTRE LA ACADEMIA Y LA POLÍTICA
}

\author{
HEALTH ECONOMIC EVALUATIONS: BRINGING \\ TOGETHER ACADEMIA AND POLICY
}

\author{
Edward Mezones-Holguín ${ }^{1,2,3, a}$
}

\begin{abstract}
En este editorial se describe, inicialmente, un panorama general de los diversos aspectos que han llevado a un incremento en el gasto en salud en los países, con la consiguiente necesidad de priorización en la asignación presupuestaria a nivel sanitario. Igualmente, se presenta a las Evaluaciones Económicas en Salud (EES) como herramientas útiles en los procesos de toma de decisiones, haciendo hincapié en las principales dificultades que existen para lograr el desarrollo y aceptación de dichas evaluaciones. Al mismo tiempo, se da una mirada rápida a los modelos y opciones que proponen algunos paradigmas de la economía de la salud. De la misma forma, se puntualizan ciertas características actuales del sistema de salud peruano, así como acciones y sugerencias para implementar el uso de las EES en el sector. En último lugar, se presenta de modo sucinto el contenido del presente número.
\end{abstract}

Se sabe que la población mundial se incrementa progresivamente; en la actualidad somos cerca de 7 mil millones y se estima que para el año 2050 podríamos alcanzar los 10 mil millones; situación que afecta sobre todo, a los países en vías de desarrollo ${ }^{(1,2)}$. Aunque durante el siglo XX la tendencia del estado de salud ha sido positiva en todo el mundo, en la actualidad esta mejora no puede ser generalizada a todas las naciones ${ }^{(3)}$. En Latinoamérica, el incremento de la expectativa de vida, la reducción de la tasa de fecundidad y las mejoras en la atención y cobertura de salud, han producido cambios demográficos significativos que han generado nuevos escenarios epidemiológicos, ergo, una redistribución y aumento del gasto en salud (4). Asimismo, a pesar de experimentarse un crecimiento económico importante, este no es proporcional al acrecentamiento de las demandas de financiación en salud; se suma a ello el hecho de que este crecimiento económico es heterogéneo, no solo entre países sino dentro de ellos, lo cual genera e intensifica la inequidad ${ }^{(4,5)}$. Es en este contexto, que la toma de decisiones respecto a la priorización en la distribución de recursos financieros en el sector salud resulta imperativa, dependiendo en gran medida del sistema de salud y de las herramientas que se disponen para ello.

En este marco, se esperaría que una EES -clásicamente entendida como la evaluación sistemática de la eficiencia económica relativa a los programas de salud ${ }^{(6)}$ - sea de utilidad para la toma de decisiones, debido al reconocimiento como instrumento válido por parte de la academia, al incremento del número de publicaciones desde la comunidad científica y al poseer tanto medidas de efecto y costos en su análisis. No obstante, el uso de las EES es limitado, siendo empleadas, fundamentalmente, en las áreas clínica y farmacológica de los países desarrollados con alta proporción de financiamiento público y con sistemas de aseguramiento universal ${ }^{(7-10)}$.

En ese sentido, si bien Latinoamérica experimenta un incremento en la producción de EES - definida como notas técnicas y publicaciones en revistas indizadas -, subyace el problema de que una proporción importante de los estudios publicados tienen limitaciones o errores metodológicos ${ }^{(11,12)}$. Del mismo modo, en muchas oportunidades no se cuenta con evidencia primaria, debido a que se adolece de falta de registros estandarizados y de buena calidad. Ambas condiciones, suponen un problema mayor, puesto que si bien

\footnotetext{
1 Unidad de Análisis y Generación de Evidencias en Salud Pública, Centro Nacional de Salud Pública, Instituto Nacional de Salud. Lima, Perú

2 Escuela de Medicina, Universidad Peruana de Ciencias Aplicadas. Lima, Perú.

3 Facultad de Medicina Humana, Universidad Nacional de Piura. Piura, Perú

a Médico, Magíster en Epidemiología Clínica
} 
existen estudios económicos desarrollados en naciones de primer mundo, estos pueden ser solo referenciales mas no generalizables a nuestra realidad y de otros países en vías de desarrollo ${ }^{(11-13)}$; debido no solo a las disimilitudes en los costos adoptados, sino a las diferencias de índole epidemiológico. Sin embargo, algunos países como Brasil, Cuba, Chile y México han realizado un mayor esfuerzo, logrando la estandarización de las pautas para la realización de EES e incorporándolas paulatinamente en la puesta en marcha de sus respectivas agendas de salud (14). Adicionalmente, como una iniciativa regional, el Organismo Andino de Salud impulsa una red de Evaluaciones de Tecnologías Sanitarias (ETS), conformada por sus países miembros, que incluye dentro de sus objetivos el desarrollo de EES.

Se han descrito múltiples barreras para la elaboración, implementación y aceptación de las EES en el proceso de toma de decisiones, situación de mayor complejidad en el ámbito de la Salud Pública, donde la priorización para la adjudicación de recursos, requiere un abordaje poblacional que supera la mirada de la eficiencia asignativa. Es así que entran a tallar consideraciones como la equidad, la afectación y flexibilidad presupuestaria, las preferencias culturales, las expectativas sociales, las orientaciones intersectoriales, las consideraciones éticas, entre otras ${ }^{(7,8,10,15)}$. Asimismo, es preciso remarcar dos de las limitantes cardinales en el proceso: primero, el "divorcio" que suele ocurrir entre los tomadores de decisiones (paradigma político) y los investigadores (paradigma científico) ${ }^{(6)}$, que es acentuado por las carencias de conocimiento y habilidades técnicas en ambos grupos, inclusive en países desarrollados ${ }^{(8,9,11,12)}$; y, segundo, generalmente el tiempo necesario para llevar a cabo una EES es superior al requerido por los decisores, por lo que su pertinencia se ve debilitada, siendo frecuente que se tengan conclusiones cuando ya las decisiones han sido tomadas $(7,10,11)$.

Por otro lado, si lo miramos desde un enfoque principista de la Economía de la Salud, todo lo expuesto hasta ahora estaría en el marco de la economía normativa o la economía de "lo que debe de ser"; puesto que emite juicios de valor que pueden tener un ingrediente ético $y$ moral, que no pueden demostrarse que sean verdaderos o falsos en el mundo real. Por lo contrario, su contraparte la economía positiva o economía de "lo que es", se centra en la descripción y la predicción de las relaciones económicas que existen, por lo que supone en teoría que no está sometida a un juicio de valor (value free economics); produciendo factores plausibles de ser contrastados en el mundo real ${ }^{(8)}$.

El modelo normativo permite considerar directamente la información producida por las investigaciones previas, y origina una recomendación centrada en una intervención o política específica bajo indicadores mixtos de costos y efectos -acá se encuentran los estudios de EES clásicos: minimización de costos, costo-beneficio, costoefectividad y costo-utilidad. Este enfoque racional del uso de la investigación se denomina "modelo resolución de problemas" (problem solving model), pero como hemos visto, en la práctica su éxito depende substancialmente de la adopción por parte de los decisores. Es allí, donde surge el "modelo interactivo (interactive model), en el cual el punto clave es la capacidad de negociación entre gestores y científicos en todas las etapas del desarrollo de alternativas de solución para un cuestionamiento concreto. También, de ser necesario pueden incluirse las costumbres de la población con lo que se convertiría en un "modelo interactivo social" (social interactive model) ${ }^{(8)}$.

Ahora bien, en las circunstancias en las que el acuerdo entre investigadores y decisores resulta difícil, especialmente cuando existen objetivos múltiples, la "economía positiva" presenta el análisis de costo-consecuencia como un opción conveniente, que a diferencia de su contraparte normativa, no se resume en un solo indicador consolidado, sino que presenta información desagregada de costos y efectos en unidades naturales que pueden ser observables y medibles. Además, este análisis no centra su recomendación en una política o intervención en particular, sino que da la opción de elegir a los decisiones en una realidad más amplia, por lo que muchas veces son mejor aceptadas que las EES normativas ${ }^{(8)}$.

En esencia, tanto el enfoque normativo como el positivo no son mutuamente excluyentes, ambos buscan promover y consolidar una cultura de políticas públicas basadas en la evidencia, pero con técnicas distintas. El primero mediante el uso de la investigación previamente desarrollada y supuestos, como base para su análisis, formulando indicadores consolidados de costos y efectos para una intervención específica; por ejemplo: una cantidad determinada de dólares por años de vida ajustados a calidad. Por su lado, el segundo se basa fundamentalmente en la información y datos reales, presentando indicadores separados y no dirigidos a una intervención en particular, por lo que puede dar medidas de impacto más directamente evaluables como el número de horas hombre ahorradas. El inclinarse por una opción u otra va a depender de las circunstancias que se enfrenten y de los objetivos que se persiguen, así como, del compromiso y concertación tanto de investigadores como políticos.

Todo lo expuesto previamente cobra alta importancia en Perú, debido a que el crecimiento económico sostenido 
en la última década contrasta con la acentuación de la inequidad; igualmente, el financiamiento público, aunque claramente limitado, sigue siendo el más importante en el sector salud, donde se lidia con los cambios graduales y cada vez más marcados del perfil epidemiológico. Del mismo modo, se viene implementado el proceso de aseguramiento universal por parte del Ministerio de Salud (MINSA) y se atraviesa por un proceso de descentralización de la salud, que busca una mayor participación de los gobiernos regionales y municipales y, al mismo tiempo, son varias las intervenciones posibles en el campo sanitario que están en el marco del Presupuesto por Resultados impulsado desde el Ministerio de Economía y Finanzas.

Bajo estas circunstancias, se requiere un proceso informado y sólido de toma de decisiones en salud a todo nivel, que permita el mejor direccionamiento y priorización de los recursos económicos pero que, a su vez, logren impacto a nivel poblacional. En este contexto, la aproximación con EES es relevante, sobre todo en el sector público, donde ya se están dando iniciativas -aunque desde la perspectiva más amplia que propone la Salud Pública Basada en Evidencias- con productos técnicos que han servido para orientar la toma de decisiones en el sector, como es el caso de lo desarrollado por el Instituto Nacional de Salud en áreas tan diversas como inmunizaciones, nutrición o tratamientos antivirales. Sin embargo, estas actividades requieren de una voluntad política que busque su reforzamiento, potenciación y reconocimiento.

Tras lo sintetizado, nace la exigencia de una estrategia de desarrollo y motivación de capacidades de los recursos humanos en EES, con la correspondiente promoción y despliegue del trabajo en equipo enfatizando en la comunicación como uno de los elementos primordiales para lograr el acoplamiento requerido de la investigación en la política ${ }^{(10,12,16)} \mathrm{Al}$ mismo tiempo, se sugiere la incorporación de otros actores clave en el proceso, por medio del análisis de partes interesadas -por ejemplo, la inclusión de los potenciales usuarios de las intervenciones- y las técnicas de priorización multicriterio, entre otros métodos. Resulta también imprescindible generar indicadores de efectividad propios del país; por lo que debería de facilitarse y promoverse el desarrollo de investigaciones piloto, ensayos clínicos comunitarios y pragmáticos que permitan obtenerlos; así como, el mejoramiento de los sistemas de registro y vigilancia epidemiológica. Además, para el futuro desarrollo de estudios de costo utilidad que tienen a la calidad de vida como medición medular, se requiere de aproximaciones lo más cercanas posibles a nuestra realidad, por lo que para su obtención, el desarrollo de encuestas poblacionales es una opción accesible que sería de gran ayuda.
Finalmente, la Revista Peruana de Medicina Experimental y Salud Publica, en su función como ente de difusión científica y académica en Salud Pública, ha reconocido como prioritario el desarrollo del presente número destinado a las Evaluaciones Económicas en Salud (EES), con el objetivo de brindar un alcance general de los aspectos metodológicos, implicaciones, ventajas y limitaciones. En este número se incluye estudios económicos peruanos en temas de alta relevancia como la vacunación para el virus del papiloma humano, la tuberculosis y la enfermedad renal crónica. Asimismo, Loza et al. llevan a cabo una revisión de los conceptos más importantes para lograr entender a las EES. En el simposio, en primera instancia, Bolaños-Díaz et al. exponen la importancia de la necesidad de la evaluación de la calidad de los estudios de síntesis como base para una EES, así mismo, Espinoza et al. formulan una propuesta de implementación de las EES para América Latina, luego Garay et al plantean un modelo para el análisis de impacto presupuestario en salud y, por último, Tolentino Silva comparte la experiencia en ETS del Ministerio de Salud de Brasil. Con lo que esperamos contribuir a mejorar el nivel de conocimiento de las EES en los procesos de toma de decisiones, así como, generar conciencia de su versatilidad y aplicabilidad en distintas esferas de la salud.

\section{REFERENCIAS BIBLIOGRÁFICAS}

1. Lee R. The outlook for population growth. Science. 2009; 333(6042):569-73.

2. Wolfgang L, Samir KC. Dimensions of global population projections: what do we know about future population trends and structures? Philos Trans R Soc Lond B Biol Sci. 2010; 365(1554): 2779-2791.

3. McMichael AJ, McKee M , Shkolnikov V ,Valkonen T. Mortality trends and setbacks: global convergence or divergence? Lancet 2004 ; 363 (9415): 1155 - 59 .

4. Lu C, Schneider MT, Gubbins P, Leach-Kemon K, Jamison D, Murray CJ. Public financing of health in developing countries: a cross-national systematic analysis. Lancet. 2010; 375(9723):1375-87.

5. Blackman T. Public spending and life expectancy. Maturitas 70 (2011) 95- 6.

6. Drummond MF, O'Brien BJ, Stoddart GL, TorranceGW. Methods for the economic evaluation of health care programs. Oxford Medical Publications; 2005. (8)

7. Chisholm D, Evans D. Economic evaluation in health: saving money or improving care? Journal of Med Economics 2007; 10: 325-37

8. Williams I, Bryan S. Understanding the limited impact of economic evaluation in health care resource allocation: A conceptual framework. Health Policy. 2007; 80(1): 135-143.

9. Eddama O, Coast J. A systematic review of the use of economic evaluation in local decision-making. Health Policy. 200886 (2-3): 129-41 
10. Augustovski F, Iglesias C, Manca A, Drummond M, Rubinstein A, Martí SG. Barriers to generalizability of health economic evaluations in Latin America and the Caribbean region. Pharmacoeconomics. 2009; 27(11):919-29.

11. Weatherly H, Drummond M, Claxton $K$, Cookson R, Ferguson B, Godfrey C, et al. Methods for assessing the costeffectiveness of public health interventions: Key challenges and recommendations. Health Policy. 2009; 93(2-3): 85-92

12. Iglesias CP, Drummond MF, Rovira J; NEVALAT Project Group. Health-care decision-making processes in Latin America: problems and prospects for the use of economic evaluation. Int J Technol Assess Health Care. 2005; 21(1):1-14.
13. Yothasamut $J$, Tantivess $S$, Teerawattananon $Y$. Using economic evaluation in policy decision-making in Asian countries: mission impossible or mission probable? Value Health. 2009;12 Suppl 3:S26-30.

14. Augustovski F, Garay OU, Pichon-Riviere A, Rubinstein A, Caporale JE. Economic evaluation guidelines in Latin America: a current snapshot. Expert Rev Pharmacoecon Outcomes Res. 2010;10(5):525-37.

15. Coast J. Is economic evaluation in touch with society's health values? BMJ 2004;329(7476):1233-6.

16. Fiestas F. Traducción de la investigación en acciones específicas en salud. Rev Per Med Exp Salud Publica. 2010; 27(3): 478-9.

\section{Consulte la versión electrónica de la Revista Peruana de Medicina Experimental y Salud Pública en www.pubmed.gov}

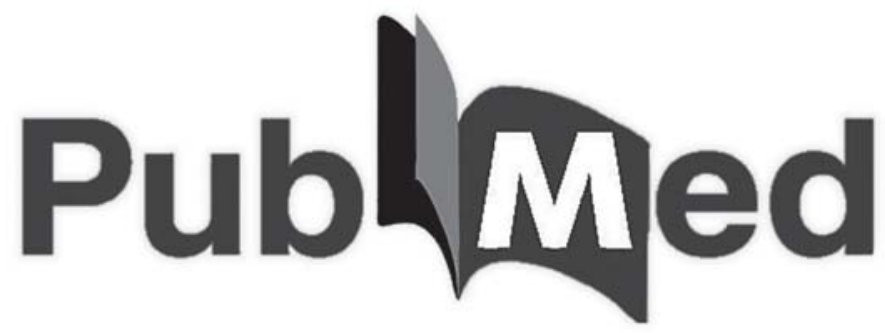

